\title{
Rapid diagnosis of Propionibacterium acnes infection in patient with hyperpyrexia after hematopoietic stem cell transplantation by next-generation sequencing: a case report
}

Mingzhi Ye ${ }^{1,2+}$, Wei Wei $^{1+}$, Zhikai Yang ${ }^{1 \dagger}$, Yingzhen $\mathrm{Li}^{1}$, Shaomin Cheng ${ }^{1}$, Kang Wang ${ }^{1}$, Tianliangwen Zhou', Jingmeng Sun ${ }^{1}$, Sha $\mathrm{Liu}^{3}, \mathrm{Na} \mathrm{Ni}^{1}$, Hui Jiang ${ }^{4^{*}}$ and Hua Jiang ${ }^{3^{*}}$

\begin{abstract}
Background: The rapid determination of pathogenic agent is very important to clinician for guiding their clinical medication. However, current diagnostic methods are of limitation in many aspects, such as detecting range, time-consuming, specificity and sensitivity. In this report, we apply our new-developing pathogen detection method to clarify that Propionibacterium acnes is the causative agent of a two-year-old boy with juvenile myelomonocytic leukemia presenting clinical symptoms including serious rash and hyperpyrexia while traditional clinical methods of diagnosis fail to detect the pathogenic agent and multiple antimicrobial drugs are almost ineffective Propionibacterium acnes is confirmed to be the infectious agent by quantitative real-time polymerase chain reaction.

Case presentation: After haploidentical hematopoietic stem cell transplantation, a two-year-old boy with juvenile myelomonocytic leukemia presented to a pediatrist in a medical facility with hyperpyrexia and red skin rash which later changed to black skin rash all over his body. Traditional diagnostic assays were unrevealing, and several routine antimicrobial treatments were ineffective, including the vancomycin, meropenem, tobramycin, cefepime and rifampin. In this case, pediatrist resorted to the next-generation sequencing technology for uncovering potential pathogens so as to direct their use of specific drugs against pathogenic bacteria. Therefore, based on the BGISEQ100 (lon Proton System) which performed sequencing-by-synthesis, with electrochemical detection of synthesis, and each such reaction coupled to its own sensor, which are in turn organized into a massively parallel sensor array on a complementary metal-oxidesemiconductor chip, we detect and identify the potential pathogens. As a result, we detected a significantly higher abundance of skin bacteria Propionibacterium acnes in patient's blood than controls. It had been reported that patients infected by Propionibacterium acnes almost always had history of immunodeficiency, trauma or surgery. Considering this possible cause, antimicrobial treatment was adjusted to target this rare opportunistic pathogen. Fever and black skin rashes were rapidly reduced after administrating specific drugs against Propionibacterium acnes.
\end{abstract}

Conclusion: This case showed our new-developing pathogen detection method was a powerful tool in assisting clinical diagnosis and treatment. And it should be paid more attention to Propionibacterium acnes infection in clinical cases.

Keywords: Propionibacterium acnes, Hyperpyrexia, Hematopoietic stem cell transplantation, Next generation sequencing, Diagnosis

\footnotetext{
*Correspondence: jianghui@genomics.cn; weimeitiantang1213@163.com

${ }^{\dagger}$ Equal contributors

${ }^{4}$ BGI-Shenzhen, Shenzhen 518083, China

${ }^{3}$ Hematopoietic Stem Cell Transplant Center, Guangzhou Women and

Children Medical Center, Guangzhou 510000, China

Full list of author information is available at the end of the article
} 


\section{Background}

Propionibacterium acnes (P. acnes) is a skin commensal bacterium. On rare occasions, it can cause serious postoperative complications, such as infective endocarditis, thrombophlebitis, and acute suppurative pericarditis, especially in immunodeficiency patients who are highly susceptible to pathogenic microorganisms. And these infections often run an acute course due to the weak or late inflammatory response [1], but their clinical symptoms are usually atypical, making them very difficult to diagnose. Till now, there have been various pathogendetecting methods applied in clinic, such as isolation and culture, serological test, specific polymerase chain reaction (PCR) and its derivatives and so on, but all these methods are almost always based on the known sequences or components and usually target one or several major known pathogens, presenting serious limitations [2]. As the development of sequencing technology with its cost continually falling, next-generation sequencing (NGS) has become an attractive tool for broad-based pathogen discovery. NGS have strong potential to detect and identify almost varieties of microorganisms, including known and unknown. In 2008, Palacios G et al. identified a new virus from three patients who received visceral-organ transplants from a single donor by high-throughput sequencing, showing a powerful tool for discovery of new pathogens [3]. In 2013, a 14-year-old boy was enrolled for pathogen detection by use of NGS because of the failure of traditional diagnostic assays, resulting ultimately in a favorable outcome [4],which indicated that the pathogen detection method based on NGS could be useful in clinical cases, Recently, a semiconducting sequencing platform (BGISEQ100) has been improved greatly, possessing some remarkable technique features, such as fast (sequencing within 2-3 h), flexible (flexible scaled chips for different throughput needs) and high accuracy (99.97\%). Here, we utilize this platform to develop a pathogen detection method for discovering the potential pathogens of unknown infection, successfully identifying an opportunistic pathogen ( $P$. acnes) that may be the major cause of serious infection of a two-year-old boy. Our result shows a reliable method for detecting potential pathogens of unknown infection in HSCT patients, indicating its strong application values in clinic.

\section{Case presentation}

On January 5th, 2014, a 2-year-old boy with JMML was presented to the pediatric hematology and oncology department for abnormal hemogram lasting for two months. Then, he was admitted to the hospital and discharged 8 days later after completing the Human Leukocyte Antigens (HLA) matching (Fig. 1a). Subsequently, he continued with chemotherapy and outpatient medications, including mercaptopurine, prednisone, and 13-cis-retinoid acid (isotretinoin).

On June 5th, 2014, the patient returned to the hospital for preparation before HSCT. His vital signs were normal, and physical examination was unremarkable except some discrete old rashes on skin (Fig. 2). The result of hospital laboratory examinations was also normal. Since June 10th, patient started to receive a myeloablative conditioning regimen [busulfan (BU) $1.2 \mathrm{mg} / \mathrm{kg}$ iv. q6h for 4 days, cyclophosphamide (CTX) $50 \mathrm{mg} / \mathrm{kg}$ iv. qd for 4 days and anti-thymocyte globulin (ATG) $3.3 \mathrm{mg} / \mathrm{kg}$ iv. qd for 3 days]. However, the patient suffered from fever (up to $39.8{ }^{\circ} \mathrm{C}$ ) with mild cough and running nose since June 15 th, indicating respiratory infection. Subsequently, clinical examinations were performed to analyze the possible cause. The blood/marrow culture were negative. The number of his peripheral white blood cells and neutrophils was 7500 and 2450 per cubic millimeter, respectively. The C-reactive protein (CRP) was above $200 \mathrm{mg}$ per liter (normal range, 0 to $5 \mathrm{mg}$ per liter). The procalcitonin (PCT) was $0.15 \mathrm{ng}$ per milliliter (normal range, 0 to $0.1 \mathrm{ng}$ per milliliter). The $\mathrm{G}$ test which was target for broad spectrum dectection of fungal infection was $20.6 \mathrm{pg}$ per milliliter (normal range, 0 to 20 pg per milliliter). All these results from assays of serum liverenzyme, usea nitrogen, creatinine, electrolyte and pathogen specific antigen were within range of normal value. However, the result of chest X-ray showed increased lung-markings. Consequently, the patient was treated with meropenenm (MEM) targeted for gram-negative and other refractory bacteria, and vancomycin (VANC) targeted for gram-positive cocci. Meanwhile, antifungal agent caspofungin acetate (CAS) and antiviral agent acyclovir $(\mathrm{ACV})$ were also administered as prophylaxis (Fig. 1b).

The patient underwent the cord blood transplantation and HSCT (the donor was his mother) on June 20th and 21 th, respectively. From June 15 th to June 24th, patient's body temperature dropped to $38.5{ }^{\circ} \mathrm{C}$ from $40.5{ }^{\circ} \mathrm{C}$, then rose to $40.3{ }^{\circ} \mathrm{C}$ again (Fig. 1c). Meanwhile, CRP dropped to $8.5 \mathrm{mg}$ per liter (normal range, 0 to $5 \mathrm{mg}$ per liter).

Although treatments had been performed regularly, his symptoms of fever, chills and cough still didn't lighten. Physical examination showed some previous discrete rashes and coarse lung sounds. CRP was $119.2 \mathrm{mg}$ per liter (normal range, 0 to $5 \mathrm{mg}$ per liter). MEM and VANC almost had no effect on the clinical symptoms. Then, his primary care physician decided to switch drugs to linezolid (LZD) against gram-positive cocci, tobramycin (TOB) against gram-negative bacteria for a week, and voriconazole for fungal prophylaxis (Fig. 1b).

During this period, patient also experienced anemia and thrombocytopenia occasionally. After transfusion of washed red blood cells and platelets for skin bleeding, 


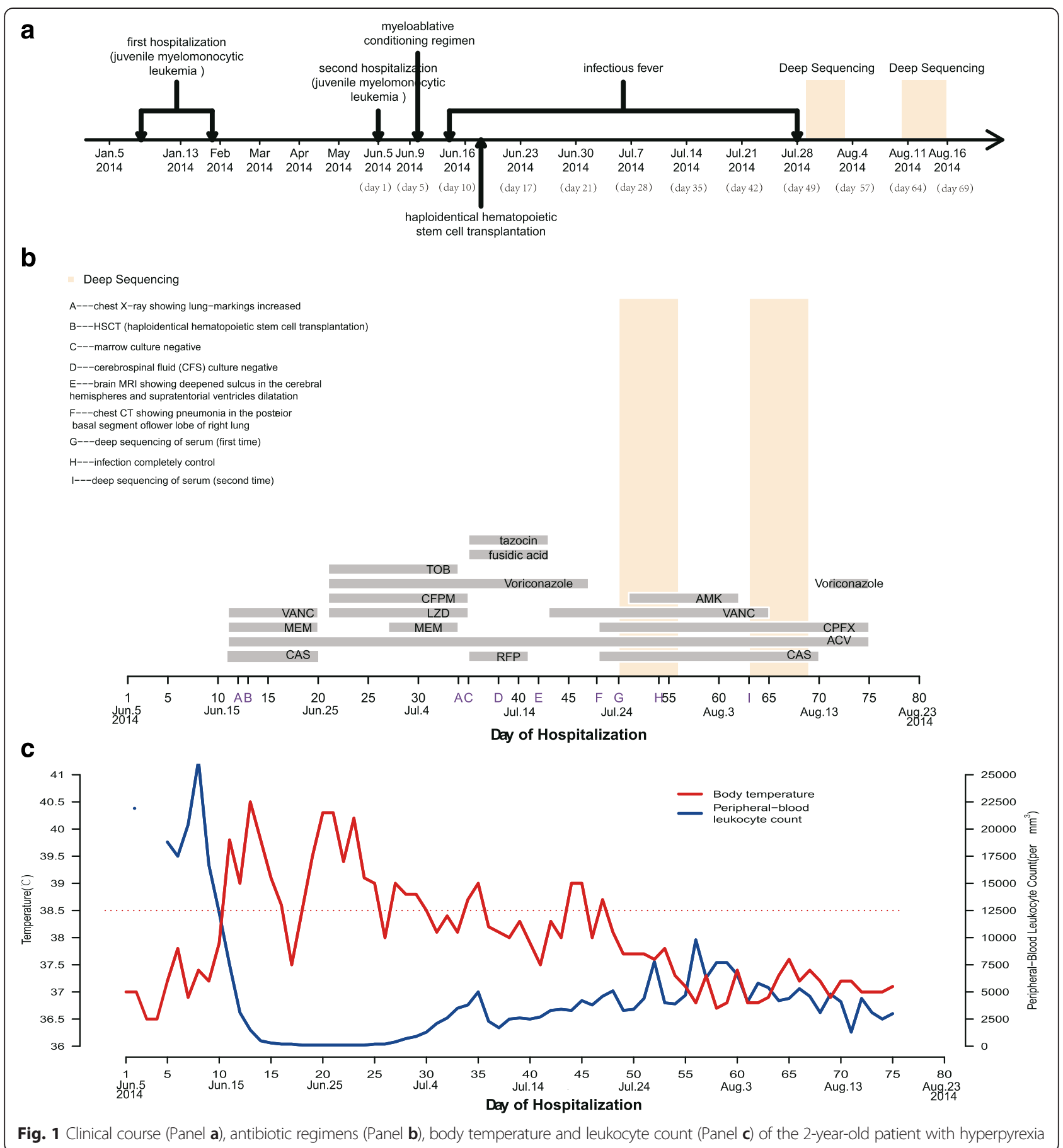

the number of his hemoglobin and platelets returned to normal. During the next two weeks, he still presented with fever, mild expectoration and some discrete old rashes, suggesting the possibility of tuberculosis bacillus infection. Considering the medication safety, the doctor stopped using TOB and LZD, and changed to use the rifampicin (RFP) and fusidic acid against gram-positive bacteria and tazocin against drug-resistance gram-negative bacteria (Fig. 1b). On July 13th, lumber puncture was performed to collect cerebrospinal fluid (CSF) for tubercle bacillus detection, but no positive results were obtained by culture and PCR based methods. After a week, the computed tomography (CT) of chest showed pneumonia in the posterior basal segment of the lower lobe of right lung (Fig. 3c/d), and CRP became $0.2 \mathrm{mg}$ per liter (normal range, 0 to $5 \mathrm{mg}$ per liter), but his body temperature still fluctuated between $37.7{ }^{\circ} \mathrm{C}$ and $39^{\circ} \mathrm{C}$. Finally, we got the informed consent of patient's mother on behalf of the 


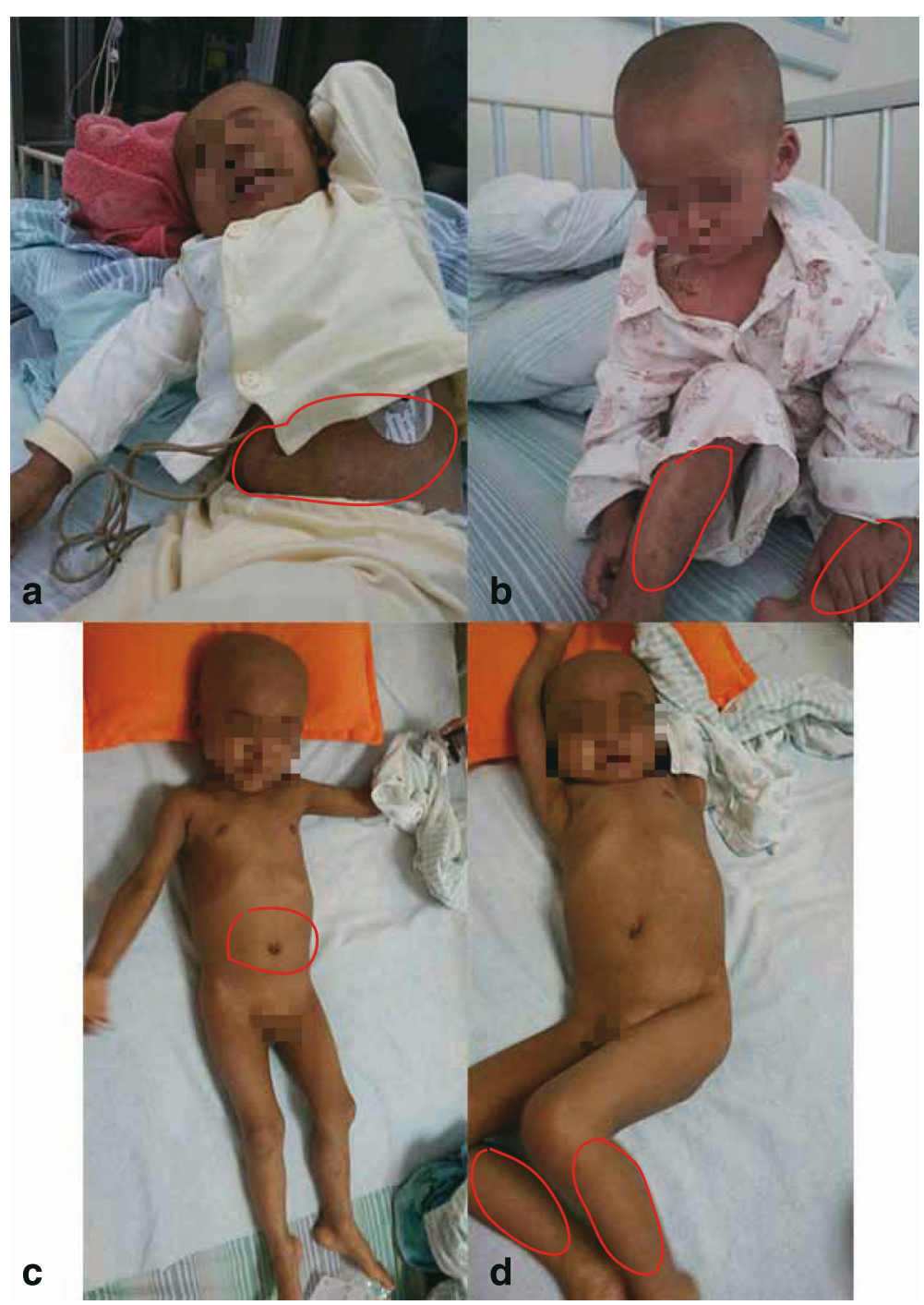

Fig. 2 The situation of skin rashes on patient's body in two time-points (pre- and post-treatment). The patient presented hyperpyrexia with black rash all over his body in the first detection (Panels $\mathbf{a}, \mathbf{b}$ ). The patient's body temperature returned to normal with the rash fading in the second detection (Panels c, d)

patient for detecting potential pathogens based on NGS technology (Fig. 1a).

Within $43 \mathrm{~h}$ after collecting patient's blood, we finished the detection of pathogens, identifying 22,123 (out of $2,602,891)$ sequence reads $(0.8499 \%)$ uniquely corresponding to Propionibacterium acnes $\left(P\right.$. acnes, $G^{+}$) genome. $P$. acnes accounted for a very high proportion in detectable microorganisms and possessed a high coverage of genome, which was also significantly higher than control (Fig. 5). This result was re-confirmed by the NGS detection and species specific real-time PCR (RT-PCR). Based on the result, the primary care physician turned to adopt specific drugs for treatment of $P$. acnes infection immediately, including VANC, ciprofloxacin (CPFX) and amikacin (AMK) against gram- positive bacteria, CAS and ACV for prophylaxis of fungal and virus infections.

Over the next 5 days, the patient gradually recovered with his body temperature returning to normal range and his old rash fading (Fig. 2c/d). However, other complications still persisted, such as the graft versus host disease (GVHD).

\section{Methods}

The NGS-based pathogen detection of patient's blood sample was approved by his parents and primary care physician. Blood samples were processed in a medical laboratory according to the Ion Torrent next-generation sequencing assay manual (BGISEQ100). The general detection process was: 1) $200 \mu \mathrm{l}$ plasma was used to 

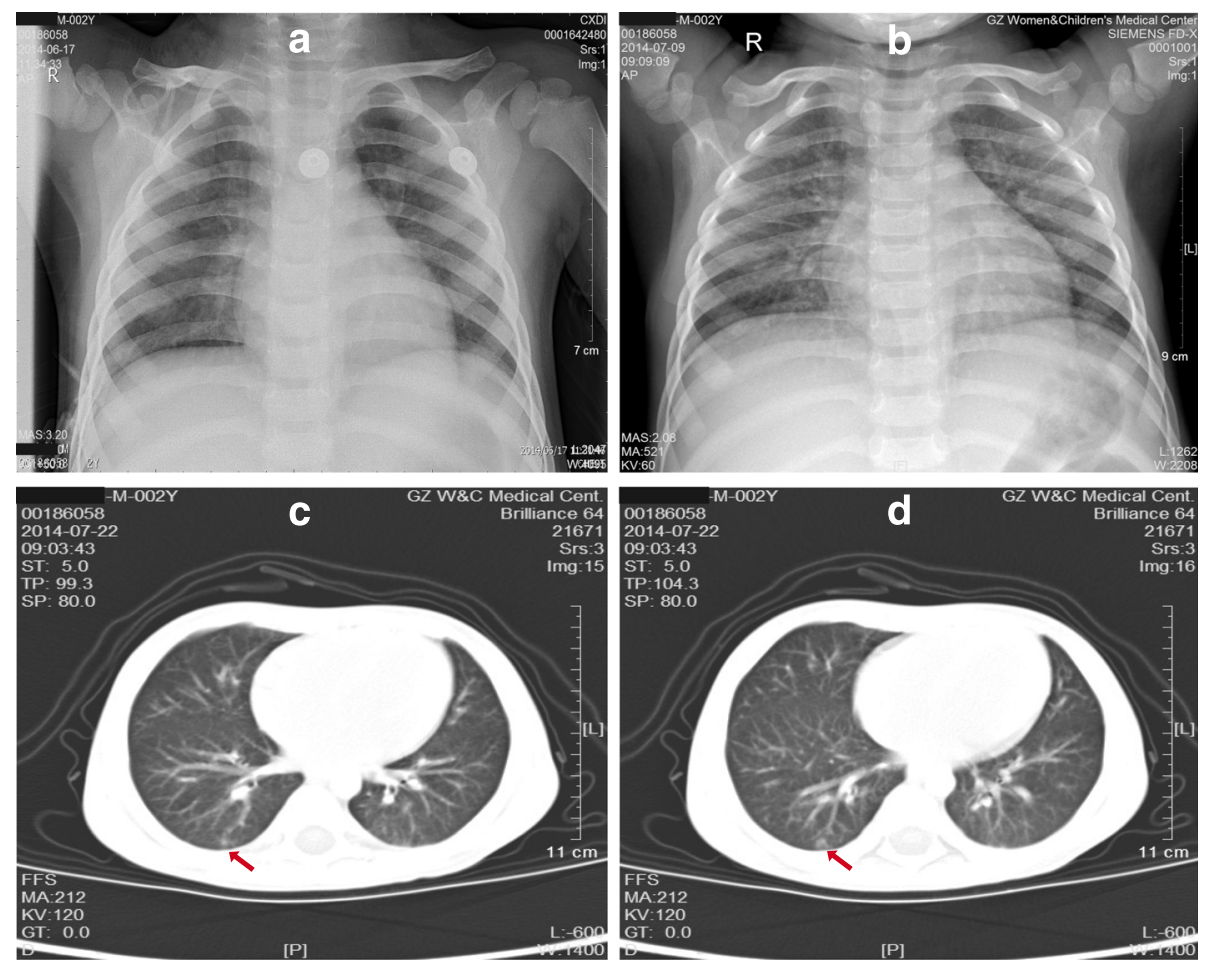

Fig. 3 The results of chest $X$-ray, CT of patient. The chest $X$-ray images showed slightly increased lung-markings from Jun 17th to July 9th (Panels $\mathbf{a}, \mathbf{b}$ ), and images of chest CT revealed pneumonia in the posterior basal segment oflower lobe of right lung on July 22nd (Panels $\mathbf{c}$, $\mathbf{d}$ )

extract nucleic acid for cDNA libraries; 2) sequencing was performed by BGISEQ100 after library was validated by 2100 Bioanalyzer system (Agilent Technologies, Inc.) and RT-PCR. Sequence reads were classified according to their origin by a bioinformatics pipeline developed by BGI (Fig. 4). The bioinformatics process mainly included the following steps: 1) host reads were subtracted; 2) remained reads were aligned to reference database, composed of multiple public sequence resources of bacteria, viruses and fungi. And the reference database was constructed according to the following steps:

1) downloaded all complete genome sequences of Bacteria, Virus from NCBI ftp site ftp://ftp.ncbi. nih.gov/genomes/;

2) removed the plasmid sequence and any other non-human-related sequence;

3) kept only one best genome sequence representing every species based on the assembly result;

4) built the alignment index of reference sequence for classing sequence reads.

In order to validate the result of NGS-based detection,specific primers were designed to detect the $P$. acnes in samples by RT-PCR. In order to accurately compare the relative abundance difference of P.acnes among different samples, human beta-actin was used as the internal reference. The designed primers were listed as:

KPA171202 of P.acnes (PA-F:5'-GCGTGAGTGACG GTAATGGGTA-3' and PA-R:5'-TTCCG ACGCGAT CAACCA-3'), and Beta-actin of human ( $\beta A-F: 5^{\prime}-A A C$ GGCTACCACATCCAAGG-3' and $\beta A-R: 5^{\prime}$-ACCAGAC TTGCCCTCCAATG-3').

\section{Results}

Rapid identification of $P$. acnes sequences in blood plasma

In detection of each sample, we selected a control sample from a non-infected patient in the same ward to do the same detection.In all of the four samples, the RNA of each sample was extracted to construct the cDNA library for sequencing. As the sequencing result, the number of reads from cDNA library of patient's plasma was $2,602,891$, and that of control was $4,294,544$. As the result of pathogen detection, $P$. acnes was identified as the most predominant pathogen taking up $0.85 \%(22,123$ out of $2,602,891$ ) of total sequence reads, $19.98 \%$ of total bacterial reads and $65 \%$ coverage of $P$. acnes genome in the first detection but reduced to $0.0048 \%$ (350 out of 7,250,976 reads) of total sequence reads, $0.43 \%$ of bacterial reads and $1.5 \%$ coverage of $P$. acnes genome in the second detection after specific drug treatments, 


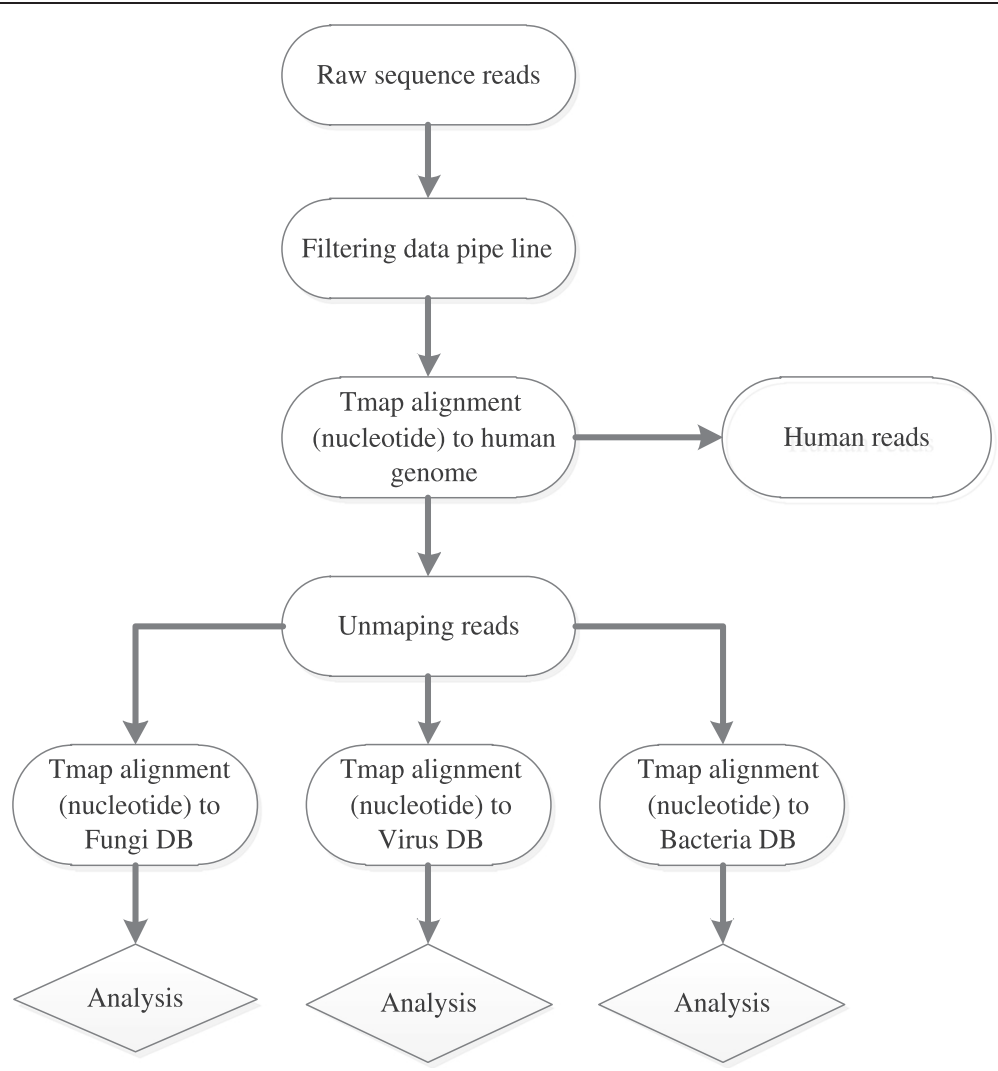

Fig. 4 The schematic overview of BGI analysis pipeline

approximate to the number of $P$. acnes reads in control (Fig. 5 and Table 1).

\section{Confirmatory testing for $P$. acnes}

Identification of $P$. acnes in patient's plasma was confirmed by RT-PCR targeted the specific gene KPA171202.As the RT-PCR result, it was no statistically significant $(P=0.56$, Student's $t$-test) of the negative control in two detections, but the patient was higher $(P=0.0056)$ than negative in the first detection and it was significantly reduced $(P=0.00094)$ in the second detection (Fig. 6), which showed a high consistency with the NGS-based result.

\section{Discussion}

P. acnes is a typical gram positive, anaerobic bacterium, which belongs to the normal skin microbiota, usually colonize skin surface and closely link with skin acne. However, its potential role in clinical infection is often underestimated due to its low virulence [5]. Furthermore, $P$. acnes is rarely diagnosed to be the major pathogen leading to serious infection not only for its pathogenicity but also methology. In aspect of clinical pathogen detection, the most common method is the culture-based method, but it possesses very poor sensitivity and accuracy and often needs to consume a large amount of time $(>7 \mathrm{~d})$ to grow microbe for analysis. Other traditional detection methods are also becoming more and more of limitation due to continual variation of microorganisms, emergence of new pathogens and flaw of method itself such as low accuracy, poor specificity and so on. Therefore, it is highly necessary to develop or utilize new detection methods to cope with these limitations. The NGS-based pathogen detection is a new-developing method for scanning the microbial sequences in clinical samples, by which we can easily identify potential pathogens for performing specific antimicrobial treatment. In clinic, P. acnes is gradually recognized as an important factor of human opportunistic infection as the number of clinical case appears to be on the rise [6-11]. However, atypical symptoms of P. acnes infections often lead to the confusion with postoperative complications, such as prosthetic joint infection, pacemaker endocarditis, and implant-associated infections [6]. In this case, although his antibody of HIV was always negative, with nutritional support therapy against malnutrition, it is highly possible that this patient is infected by the opportunistic $P$. acnes resided in the skin dermis because of his defective immune system [12]. The significant differences of read number and genomic coverage of $P$. acnes between patient and control and pre- and posttreatment strongly indicate the infection of $P$. acnes. 


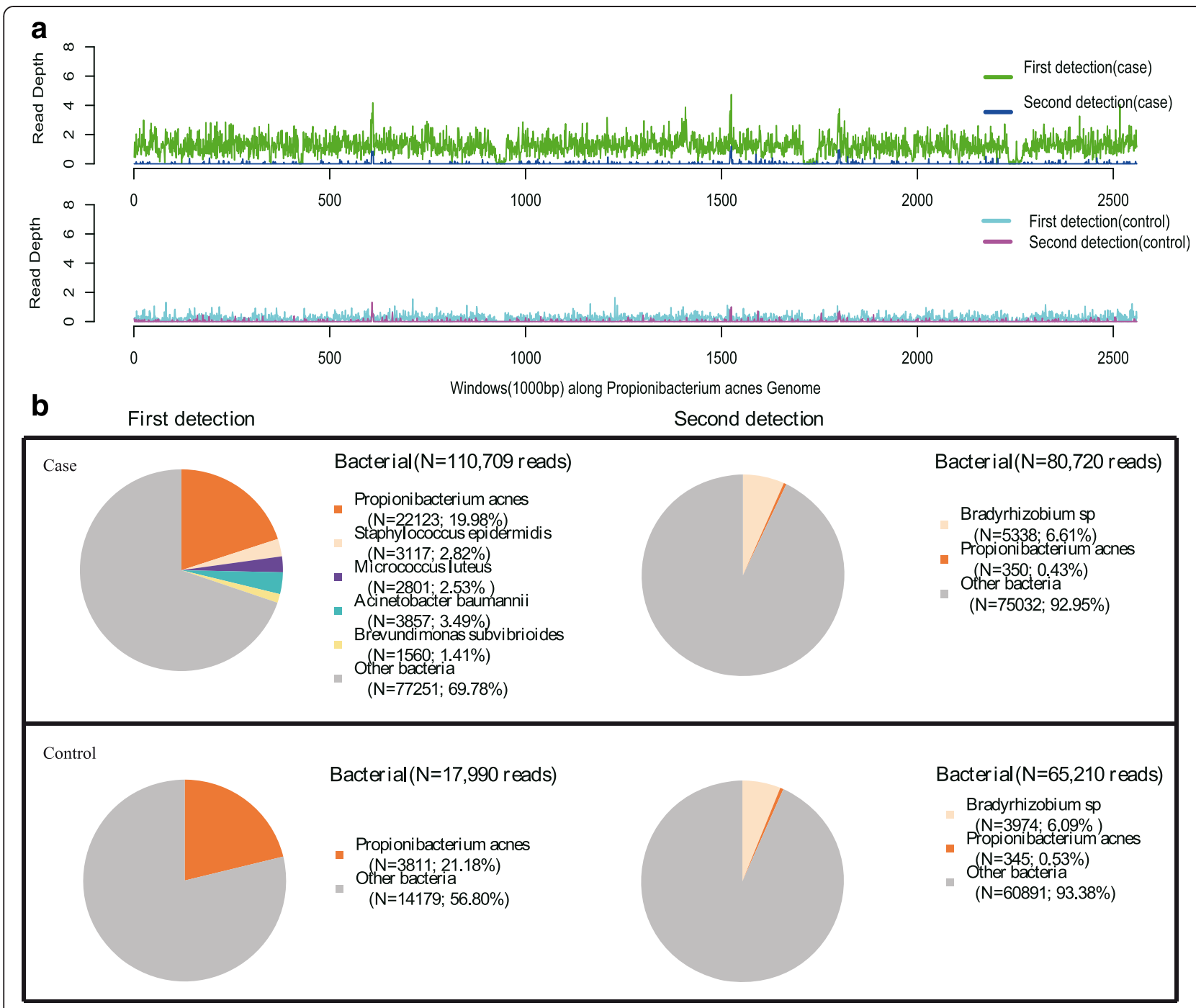

Fig. 5 Diagnosis of $P$. acnes infection by the NGS-based method: mapping of $P$. acnes reads (Panel a) and sequences in blood of two detections (Panel $\mathbf{b}$ )

Before confirming infection of $P$. acnes, Pediatricians had to choose various drugs targeting viruses, fungi, and bacteria $\left(\mathrm{G}^{+}\right.$bacterium, $\mathrm{G}^{-}$bacterium, bacillus and coccus) base on the principles of minimum damage to patients and universal prescription drug coverage. However, $P$. acnes is able to form biofilm, which renders it resistant to most antibiotics $[13,14]$, such as daptomycin and rifampin $[14,15]$. The recently published report suggests that the combination of daptomycin and rifampin, followed by levofloxacin and rifampin, might be a reasonable treatment for $P$. acnes infection [15]. Subsequently, the primary care doctor administrates drugs according to the reported dosage regimen, attaining an effective treatment. Additionally, biofilm formation is essential for resistance of some bacteria to drug. P. acnes has been shown to be able to form biofilm both in vitro and in vivo [16], which also highlights the important influence of biofilm in treatment of $P$. acnes infection [17].

Table 1 The basic situation of $P$. acnes in twice NGS-based detections

\begin{tabular}{lllll}
\hline Item & $\begin{array}{l}\text { Reads number } \\
\text { of P. acnes }\end{array}$ & $\begin{array}{l}\text { Proportion of } P \text {. acnes } \\
\text { reads in total reads }\end{array}$ & $\begin{array}{l}\text { Coverage of } P \text {. acnes } \\
\text { genome }\end{array}$ & $\begin{array}{l}\text { Depth of } P \text {. acnes } \\
\text { genome }\end{array}$ \\
\hline First detection (case) & 22,123 & $0.8499 \%$ & $65.00 \%$ & 1.9 \\
First detection (control) & 3,811 & $0.0887 \%$ & $17.00 \%$ & 1.2 \\
Second detection (case) & 350 & $0.0048 \%$ & $1.50 \%$ & 1.2 \\
Second detection (control) & 345 & $0.0048 \%$ & $1.40 \%$ & 1.3 \\
\hline
\end{tabular}




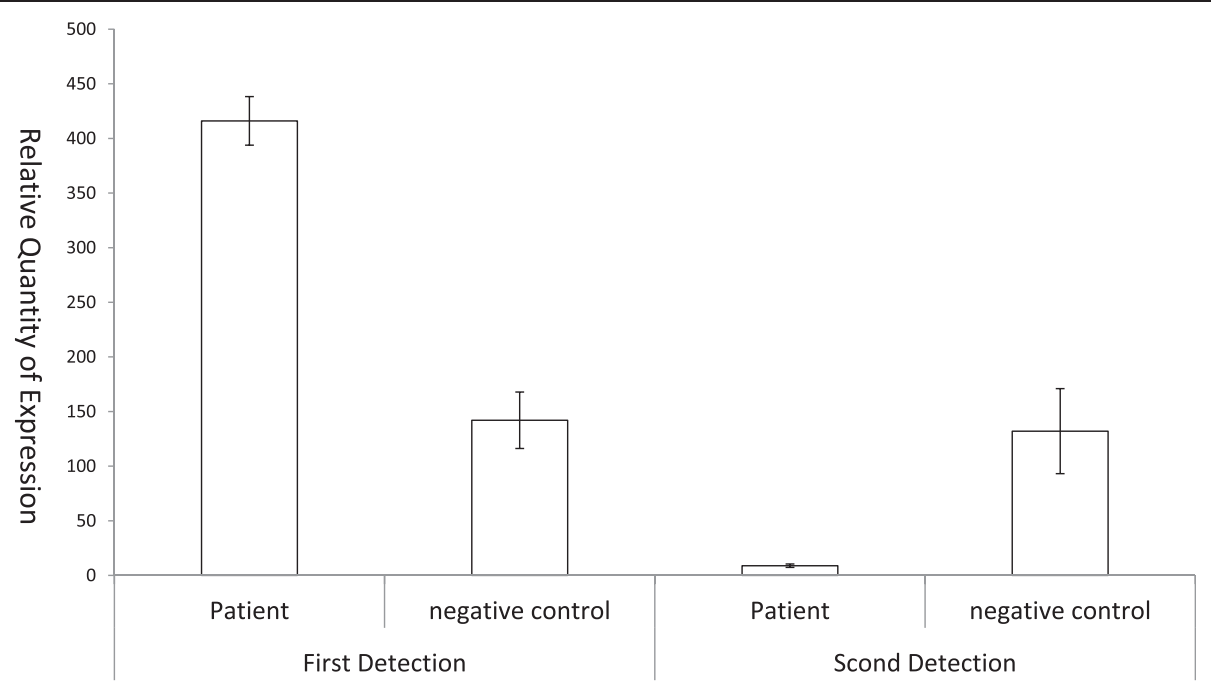

Fig. 6 Validation of P. acnes in patient's plasma by RT-PCR

\section{Conclusion}

In summary, our NGS coupled with an efficient bioinformatics pipeline successfully identified an opportunistic pathogen responsible for an infection of unknown origin, which eluded conventional assays in clinic, powerfully assisting doctors in selecting the most targeted and effective treatment for patients. The present case study also has important clinical implications, P. acnes infections may continue to increase in the patients with immuno-compromise in the future, and an optimal antimicrobial regimen needs to be defined.

\section{Consent}

Written informed consent was obtained from the patient's parent for publication of this case report. A copy of the written consent is available for review by the Editor of this journal.

\section{Abbreviations}

P. acnes: Propionibacterium acnes; PCR: Polymerase chain reaction; NGS: Next-generation sequencing; HLA: Human Leukocyte Antigens; HSCT: Haploidentical hematopoietic stem cell transplantation; JMML: Juvenile myelomonocytic leukemia; BU: Busulfan; CTX: Cyclophosphamide; ATG: Anti-thymocyte globulin; CRP: C-reactive protein; PCT: Procalcitonin; MEM: Meropenenm; VANC: Vancomycin; ACV: Antiviral agent acyclovir; LZD: Linezolid; TOB: Tobramycin; RFP: Rifampicin; CSF: Cerebrospinal fluid; CT: Computed tomography; RT-PCR: Real-time PCR; CPFX: Ciprofloxacin; AMK: Amikacin; CAS: Caspofungin acetate; GVHD: Graft versus host disease.

\section{Competing interests}

The authors declare that they have no competing interests.

\section{Authors' contributions}

All authors have reviewed and approved the manuscript. Additionally, all authors have contributed significantly to this work. ZKY, YZL and KW evaluated the detection. SMC and TLWZ performed the bioinformatics analysis. MZY and WW participated in the design of the study. WW and SL collated the patient's data. WW and JMS drafted the manuscript. NN, HuiJ and HuaJ revised the manuscript.

\section{Acknowledgments}

This work was supported by Guangzhou Key Laboratory of Cancer Trans-Omics Research(GZ2012, NO348) and ShenZhen Engineering Laboratory for Clinical molecular diagnostic.

\section{Author details}

${ }^{1}$ BGI-Guangdong, BGl-Shenzhen, Guangzhou 510006, China. ${ }^{2}$ BGl-Guangzhou, Guangzhou Key Laboratory of Cancer Trans-Omics Research, Guangzhou, China. ${ }^{3}$ Hematopoietic Stem Cell Transplant Center, Guangzhou Women and Children Medical Center, Guangzhou 510000, China. ${ }^{4}$ BGI-Shenzhen,

Shenzhen 518083, China.

Received: 10 July 2015 Accepted: 1 December 2015

Published online: 08 January 2016

\section{References}

1. Picard C, Casanova $\mathrm{J}$, Puel A. Infectious diseases in patients with IRAK-4, MyD88, NEMO, or I Badeficiency. Clin Microbiol Rev. 2011;24:490-7.

2. Chiu CY. Viral pathogen discovery. Curr Opin Microbiol. 2013:16:468-78.

3. Palacios G, Druce J, Du L, Tran T, Birch C, Briese T, et al. A new arenavirus in a cluster of fatal transplant-associated diseases. N Engl J Med. 2008;358(11):1204.

4. Wilson MR, Naccache SN, Samayoa E, Biagtan M, Bashir H, Yu G, et al. Actionable Diagnosis of Neuroleptospirosis by Next-Generation Sequencing. N Engl J Med. 2014;370:2408-17.

5. McDowell A, Patrick S, Dongyou L. "Propionibacterium," in Molecular Detection of Human Bacterial Pathogens. 1st ed. Orlando: CRC press, Taylor and Francis; 2011. p. 137-54.

6. Zappe B, Graf S, Ochsner PE, Zimmerli W, Sendi P. Propionibacterium spp. in prosthetic joint infections: a diagnostic challenge. Arch Orthop Trauma Surg. 2008;128:1039-46.

7. Clayton JJ, Baig W, Reynolds GW, Sandoe JA. Endocarditis caused by Propionibacterium species: a report of three cases and a review of clinical features and diagnostic difficulties. J Med Microbiol. 2006;55:981-7.

8. Arnell K, Cesarini K, Lagerqvist-Widh A, Wester T, Sjölin J. Cerebrospinal fluid shunt infections in children over a 13-year period: anaerobic cultures and comparison of clinical signs of infection with Propionibacterium acnes and with other bacteria. J Neurosurg Pediatr. 2008;1:366-72.

9. Perry A, Lambert P. Propionibacterium acnes: infection beyond the skin. Expert Rev Anti Infect Ther. 2011:9:1149-56.

10. Levy O, lyer S, Atoun E, Peter N, Hous N, Cash D, et al. Propionibacterium acnes: an underestimated etiology in the pathogenesis of osteoarthritis? J Shoulder Elbow Surg. 2013;22:505-11.

11. Santo KR, Franceschi V, Campos AC, Monteiro TS, Barbosa Gl, Dantas A, et al. Pacemaker Endocarditis Caused by Propionibacterium acnes in an Adult Patient with Ebstein's Anomaly: A Report of a Rare Case. Heart Lung Cric. 2014;23:e222-225. 
12. Lee MJ, Pottinger PS, Butler-Wu S, Bumgarner RE, Russ SM, Matsen 3rd FA. Propionibacterium persists in the skin despite standard surgical preparation. J Bone Joint Surg Am. 2014;96:1447-50.

13. Kurz M, Kaufmann BA, Baddour LM, Widmer AF. Propionibacterium acnes prosthetic valve endocarditis with abscess formation: a case report. BMC Infect Dis. 2014;14:105.

14. Achermann Y, Goldstein EJ, Coenye T, Shirtliff ME. Propionibacterium acnes: from commensal to opportunistic biofilm-associated implant pathogen. Clin Microbiol Rev. 2014;27:419-40.

15. Furustrand Tafin U, Corvec S, Betrisey B, Zimmerli W, Trampuz A. Role of rifampin against Propionibacterium acnes biofilm in vitro and in an experimental foreign-body infection model. Antimicrob Agents Chemother. 2012;56:1885-91.

16. Ramage G, Tunney MM, Patrick S, Gorman SP, Nixon JR. Formation of Propionibacterium acnes biofilms on orthopaedic biomaterials and their susceptibility to antimicrobials. Biomaterials. 2003;24:3221-7.

17. Holmberg A, Lood R, Mörgelin M, Söderquist B, Holst E, Collin M, et al, Biofilm formation by Propionibacterium acnes is a characteristic of invasive isolates. Clin Microbiol Infect. 2009:15:787-95.

\section{Submit your next manuscript to BioMed Central} and we will help you at every step:

- We accept pre-submission inquiries

- Our selector tool helps you to find the most relevant journal

- We provide round the clock customer support

- Convenient online submission

- Thorough peer review

- Inclusion in PubMed and all major indexing services

- Maximum visibility for your research 